\title{
Lectura comprensiva y pensamiento crítico de los estudiantes de la Escuela Profesional de Obstetricia de la Facultad de Ciencia Médicas de la Universidad Nacional Santiago Antúnez de Mayolo - Huaraz - 2021
}

\author{
Comprehensive reading and critical thinking of the students of the Professional School of \\ Obstetrics of the Faculty of Medical Sciences of the National University Santiago Antúnez de \\ Mayolo - Huaraz - 2021 \\ Nora Arcelia Vásquez Imac', Abelardo Rodolfo Campana Concha
}

\section{RESUMEN}

Objetivo: Conocer la relación existente entre la lectura comprensiva y el pensamiento crítico de los estudiantes. Materiales y Método: Es una investigación correlacional que evaluó dos variables, aplicándose dos cuestionarios a 140 estudiantes, para lo cual se necesitó de la aplicación de una encuesta y test de conocimiento, cuyas respuestas necesitaron del estadístico SPSS. Resultados: En la hipótesis general halló un Rho $=0.542$ que hace referencia a que un nivel regular de la lectura comprensiva se relaciona en el pensamiento crítico aplicado a veces. En los planteamientos específicos también se confirmaron relaciones entre la primera variable y las dimensiones búsqueda de la verdad, amplitud mental, capacidad de análisis, ser sistemático, confianza en la razón, curiosidad y la madurez para formular juicios. Conclusión: Existe una relación entre la lectura comprensiva (regular) y el pensamiento crítico (a veces) a nivel muy bueno que pone en evidencia que el nivel lector de los estudiantes de la muestra resulta insuficiente para desarrollar el pensamiento crítico.

Palabras clave: Lectura comprensiva, Pensamiento crítico, Amplitud mental.

\section{ABSTRACT}

Objective: To know the relationship between comprehensive reading and critical thinking of students. Materials and Method: It is a correlational investigation that evaluated two variables, applying two questionnaires to 140 students, for which it was necessary to apply a survey and knowledge test, whose responses required the SPSS statistic. Results: In the general hypothesis, he found a $R h o=0.542$ which refers to the fact that a regular level of comprehensive reading is related to critical thinking sometimes applied. In the specific approaches, relationships were also confirmed between the first variable and the dimensions search for truth, mental breadth, capacity for analysis, being systematic, confidence in reason, curiosity and maturity to formulate judgments. Conclusion: There is a relationship between comprehensive (regular) reading and critical thinking (sometimes) at a very good level that shows that the reading level of the students in the sample is insufficient to develop critical thinking.

Keywords: Comprehensive reading, Critical thinking, Wide mind.

\section{INTRODUCCIÓN}

Los resultados PISA nos permite dar cuenta de la realidad lectora en el país, ya que los estudiantes al terminar su etapa escolar evidencian problemas para comprender los textos que leen, y esto se complica aún más cuando inician sus estudios universitarios, quizá las personas que tienen un hábito lector tendrán menos problemas al leer los textos típicamente universitarios, sin embargo, es un porcentaje mínimo.

Según el Ministerio de Educación (MINEDU, 2018) en las últimas pruebas PISA, los estudiantes peruanos alcanzaron 401 puntos en la comprensión lectora, incrementándose solo 3 con respecto a las misma evaluación internacional realizada en el 2015. Sin embargo, nuestro país se encuentra ubicado muy por debajo de otros países sudamericanos como Chile que alcanzó un puntaje de 452.

Por otro lado, en la etapa universitaria, la lectura es un requisito fundamental para cumplir con las actividades académicas de los cursos, además de fortalecer el pensamiento crítico. Este punto, es compartido por Cangalaya (2020) quien considera que la universidad debe desarrollar en los estudiantes "las capacidades propias de la investigación y, con ello, el conjunto de competencias y habilidades para que puedan asumir de manera correcta las responsabilidades de su vida

\section{Recibido 16/08/2021 Aprobado 26/08/2021}

Este es un artículo de acceso abierto, distribuido bajo los términos de la Licencia Creative Commons Atribución 4.0 Internacional (http://creativecommons.org/licenses/by/4.0/)

\section{(c) (1)}

Lic. en Educación nivel primaria, Estudiante de Maestría de la Universidad Nacional Mayor de San Marcos Labora en el Colegio Nacional Parroquial Santa Rosa de Viterbo, Huaráz, Perú. Email: nvasquezi@cnpsrv.pe 
profesional y laboral".

Asimismo, es necesario puntualizar que el pensamiento crítico no solo debe tener un interés académico sino cotidiano adaptándose a las necesidades del entorno, como lo señala Pineda y Cerrón (2015):

"La capacidad de pensar críticamente es fundamental para afrontar con éxito las exigencias de la sociedad del siglo XXI y en tal sentido una de las tareas fundamentales de la educación es desarrollar esta capacidad" (p.106).

Del mismo modo, estudios anteriores nos permite comprender este contexto investigativo, Córdova (2019) considera necesario incentivar sistemáticamente el uso de estrategias metodológicas durante los momentos de lectura (antes, durante y después). De otro lado, tenemos a Mendoza (2015) encontró que la investigación influye positivamente en el pensamiento crítico ayudando a realizar un análisis más complejo sobre un determinado tema.

Por ende, el objetivo de estudio es conocer la relación existente entre la lectura comprensiva y el pensamiento crítico de los estudiantes de la escuela profesional de Obstetricia de la Facultad de Ciencia Médicas de la Universidad Nacional Santiago Antúnez de Mayolo Huaraz-2021.

\section{MÉTODO}

Es un estudio correlacional con enfoque cuantitativo, nivel explicativo, ex post facto, puesto que intenta analizar y evaluar la relación entre las dos variables presentadas. La población es de 394 estudiantes, de los cuales 140 integraron la muestra. Se les aplicó una encuesta y un test de conocimiento, luego procesado mediante el programa estadístico SPSS.

\section{RESULTADOS}

\section{Tabla 1}

Frecuencias variable lectura comprensiva

\begin{tabular}{llrr}
\hline & & Frecuencia & Porcentaje \\
\hline \multirow{4}{*}{ Válidos } & Mala & 4 & 2,9 \\
& Regular & 104 & 74,3 \\
& Buena & 30 & 21,4 \\
& Muy buena & 2 & 1,4 \\
\cline { 2 - 4 } & Total & 140 & 100,0 \\
\hline
\end{tabular}

Fuente: Nora Vásquez (2021)

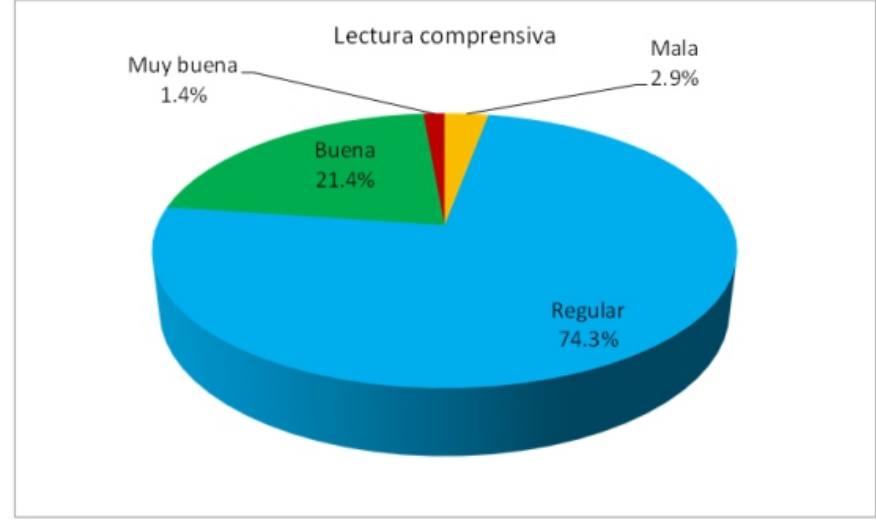

Figura 1. Porcentajes lectura comprensiva

El 74,3\% de los estudiantes alcanza un nivel regular en la comprensión lectora, el $21,4 \%$ de buena, $2,9 \%$ mala y $1.4 \%$ muy buena con ello entendemos que la mayoría de los estudiantes tienes dificultades para alcanzar el nivel académico en esta materia.

\section{Tabla 2}

Frecuencias pensamiento crítico

\begin{tabular}{rlrr}
\hline & & Frecuencia & \multicolumn{2}{c}{ Porcentaje } \\
\hline \multirow{4}{*}{ Válidos } & Casi nunca & 2 & 1,4 \\
& A veces & 103 & 73,6 \\
& Casi siempre & 33 & 23,6 \\
& Siempre & 2 & 1,4 \\
\cline { 2 - 4 } & Total & 140 & 100,0 \\
\hline
\end{tabular}

Fuente: Nora Vásquez (2021)

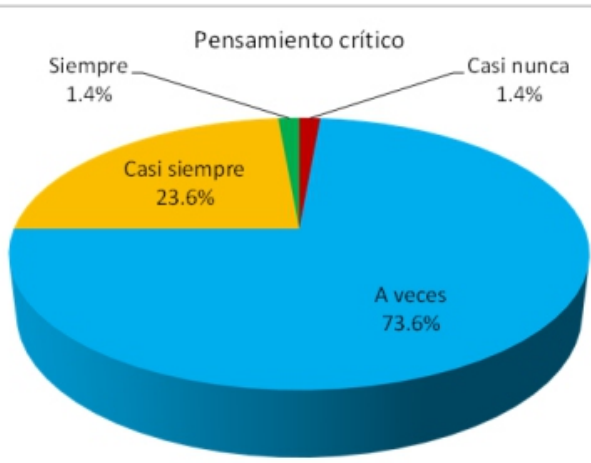

Figura 1. Porcentajes pensamiento crítico

El 73,6\% de los estudiantes considera que a veces aplica el pensamiento crítico, el $23,6 \%$ casi siempre, $1,4 \%$ siempre y $1,4 \%$ casi nunca, es decir la muestra aplica este tipo de conocimiento regularmente.

Hipótesis general

HGA. La lectura comprensiva se relaciona significativamente con el pensamiento crítico

HGO. La lectura comprensiva no se relaciona significativamente con el pensamiento crítico. 


\section{Tabla 3}

Correlación hipótesis general

\begin{tabular}{lllr}
\hline & \multicolumn{2}{c}{ Correlación } & $\begin{array}{c}\text { Pensamiento } \\
\text { crítico }\end{array}$ \\
\hline \multirow{3}{*}{$\begin{array}{l}\text { Rho de } \\
\text { Spearman }\end{array}$} & Lectura & Coeficiente de & 0,902 \\
& comprensiva & correlación & \\
\cline { 3 - 4 } & & Sig. (bilateral) & 0,000 \\
\cline { 2 - 3 } & $\mathrm{N}$ & 140 \\
\hline
\end{tabular}

Existe una relación muy buena de Rho $=0,902$ y un $p=$ 0,000 entre las variables planteadas, cuyos resultados se inclinan a la aceptación de la hipótesis alterna sobre la nula.

\section{Prueba de la hipótesis específica 1}

Ha. La lectura comprensiva se relaciona significativamente con la búsqueda de la verdad

Ho. La lectura comprensiva no se relaciona significativamente con la búsqueda de la verdad

\section{Tabla 4}

Correlación hipótesis especifica 1

\begin{tabular}{lllr}
\hline & Correlación & \multicolumn{2}{c}{$\begin{array}{c}\text { Búsqueda de } \\
\text { la verdad }\end{array}$} \\
\hline \multirow{2}{*}{$\begin{array}{l}\text { Rho de } \\
\text { Spearman }\end{array}$} & $\begin{array}{l}\text { Lectura } \\
\text { comprensiva }\end{array}$ & $\begin{array}{l}\text { Coeficiente de } \\
\text { correlación }\end{array}$ & 0,542 \\
\cline { 3 - 4 } & & Sig. (bilateral) & 0,000 \\
\cline { 3 - 4 } & $\mathrm{N}$ & 140 \\
\hline
\end{tabular}

Se evidencia una relación moderada de 0,542 y una significancia de 0,000 cuyas cifras nos permite la aceptación de la hipótesis alterna.

Prueba de la hipótesis específica 2

Ha. La lectura comprensiva se relaciona significativamente con la amplitud mental

Ho. La lectura comprensiva no se relaciona significativamente con la amplitud mental

\section{Tabla 5}

Correlación hipótesis específica 2

\begin{tabular}{lllr}
\hline \multirow{2}{*}{ Correlación } & \multicolumn{1}{c}{$\begin{array}{c}\text { Amplitud } \\
\text { mental }\end{array}$} \\
\hline \multirow{3}{*}{$\begin{array}{l}\text { Rho de } \\
\text { Spearman }\end{array}$} & $\begin{array}{l}\text { Lectura } \\
\text { comprensiva }\end{array}$ & $\begin{array}{l}\text { Coeficiente de } \\
\text { correlación }\end{array}$ & 0,655 \\
\cline { 3 - 4 } & & Sig. (bilateral) & 0,000 \\
\cline { 3 - 4 } & & $\mathrm{N}$ & 140 \\
\hline
\end{tabular}

Se acepta la hipótesis alterna debido a la significancia de 0,00 y el coeficiente de 0,655 se confirma una relación buena entre la lectura comprensiva y la amplitud mental destacando por su frecuencia regular y a veces, respectivamente.
Prueba de la hipótesis específica 3

Ha. La lectura comprensiva se relaciona significativamente con la capacidad de análisis.

Ho. La lectura comprensiva no se relaciona significativamente con la capacidad de análisis.

Tabla 5.

Correlación hipótesis específica 3

\begin{tabular}{lllr}
\hline & \multicolumn{2}{c}{ Correlación } & \multicolumn{1}{c}{$\begin{array}{c}\text { Capacidad } \\
\text { de análisis }\end{array}$} \\
\hline \multirow{3}{*}{$\begin{array}{l}\text { Rho de } \\
\text { Spearman }\end{array}$} & $\begin{array}{l}\text { Lectura } \\
\text { comprensiva }\end{array}$ & $\begin{array}{l}\text { Coeficiente de } \\
\text { correlación }\end{array}$ & 0,508 \\
\cline { 3 - 4 } & & Sig. (bilateral) & 0,000 \\
\cline { 2 - 3 } & $\mathrm{N}$ & 140 \\
\hline
\end{tabular}

Se confirma la aceptación de la $\mathrm{Ha}$ debido a la significancia de 0,00 y el coeficiente de 0,508 se confirma una relación moderada entre la lectura comprensiva y la capacidad de análisis destacando por su frecuencia regular y a veces, respectivamente.

Prueba de la hipótesis específica 4

Ha. La lectura comprensiva se relaciona significativamente con ser sintético

Ho. La lectura comprensiva no se relaciona significativamente con ser sintético

Tabla 6.

Correlación hipótesis específica 4

\begin{tabular}{|c|c|c|c|}
\hline \multicolumn{2}{|c|}{ Correlación } & & $\begin{array}{c}\text { Ser } \\
\text { sistemático }\end{array}$ \\
\hline \multirow{3}{*}{$\begin{array}{l}\text { Rho de } \\
\text { Spearman }\end{array}$} & \multirow{3}{*}{$\begin{array}{l}\text { Lectura } \\
\text { comprensiva }\end{array}$} & $\begin{array}{l}\text { Coeficiente de } \\
\text { correlación }\end{array}$ & ,608 \\
\hline & & Sig. (bilateral) &, 000 \\
\hline & & $\mathrm{N}$ & 140 \\
\hline
\end{tabular}

Se identificó una relación buena de 0,608 y una significancia de 0,000 cuyos números permite rechazar el planteamiento nulo, asimismo confirma que una regular lectura comprensiva permite el desarrollo de su ser sistemático con una frecuencia de a veces.

\section{Prueba de la hipótesis específica 5}

Ha. La lectura comprensiva se relaciona significativamente con la confianza en la razón.

Ho. La lectura comprensiva no se relaciona significativamente con la confianza en la razón. 
Tabla 7

Correlación hipótesis específica 5

\begin{tabular}{lllr}
\hline & Correlación & \multicolumn{1}{c}{$\begin{array}{c}\text { Confianza en } \\
\text { la razón }\end{array}$} \\
\hline \multirow{3}{*}{$\begin{array}{l}\text { Rho de } \\
\text { Spearman }\end{array}$} & $\begin{array}{l}\text { Lectura } \\
\text { comprensiva }\end{array}$ & $\begin{array}{l}\text { Coeficiente de } \\
\text { correlación }\end{array}$ & 0,582 \\
\cline { 3 - 4 } & & Sig. (bilateral) & 0,000 \\
\cline { 3 - 4 } & $\mathrm{N}$ & 140 \\
\hline
\end{tabular}

En las estadísticas se identificó una relación moderada de 0,582 y una significancia de 0,000 cuyos números permite rechazar el planteamiento nulo, asimismo confirma que una regular lectura comprensiva permite el desarrollo de su confianza en la razón con una frecuencia de a veces.

\section{Prueba de la hipótesis específica 6}

Ha. La lectura comprensiva se relaciona significativamente con la curiosidad.

Ho. La lectura comprensiva no se relaciona significativamente con la curiosidad.

Tabla 8.

Correlación hipótesis específica 6

\begin{tabular}{lllr}
\hline \multicolumn{2}{c}{ Correlación } & \multicolumn{1}{c}{ Curiosidad } \\
\hline \multirow{3}{*}{$\begin{array}{l}\text { Rho de } \\
\text { Spearman }\end{array}$} & $\begin{array}{l}\text { Lectura } \\
\text { comprensiva }\end{array}$ & $\begin{array}{l}\text { Coeficiente de } \\
\text { correlación }\end{array}$ & $0,664^{* *}$ \\
\cline { 3 - 4 } & & Sig. (bilateral) & 0,000 \\
\cline { 3 - 4 } & $\mathrm{N}$ & 140 \\
\hline
\end{tabular}

Se da la aceptación de la hipótesis alterna debido a la significancia de 0,00 y el coeficiente de 0,664 con el cual se confirma una relación buena entre la lectura comprensiva y la curiosidad destacando por su frecuencia regular y a veces, respectivamente.

\section{Prueba de la hipótesis específica 7}

Ha. La lectura comprensiva se relaciona significativamente con la madurez para formular juicios.

Ho. La lectura comprensiva no se relaciona significativamente con la madurez para formular juicios.

Tabla 9.

Correlación hipótesis específica 7

\begin{tabular}{lllr}
\hline & Correlación & & \multicolumn{1}{c}{$\begin{array}{c}\text { Madurez para } \\
\text { formular } \\
\text { juicios }\end{array}$} \\
\hline \multirow{2}{*}{$\begin{array}{l}\text { Rho de } \\
\text { Spearman }\end{array}$} & $\begin{array}{l}\text { Lectura } \\
\text { comprensiva }\end{array}$ & $\begin{array}{l}\text { Coeficiente } \\
\text { de correlación }\end{array}$ & 0,667 \\
\cline { 3 - 4 } & Sig. (bilateral) & 0,000 \\
\cline { 3 - 4 } & $\mathrm{N}$ & 140 \\
\hline
\end{tabular}

Se evidencia una relación moderada de 0,667 y una significancia de 0,000 cuyas cifras nos permite la aceptación de la hipótesis alterna, asimismo considera que un regular dominio de la lectura comprensiva confirma que a veces aplica una madurez para formular juicios.

\section{DISCUSIÓN DE LOS RESULTADOS}

En las estadísticas se aprecia que entre la variables $\mathrm{X}$ e $\mathrm{Y}$ existe una relación muy buena $(\mathrm{Rho}=0,902)$ debido a los resultados mayoritarios en la lectura compresiva (74,3\%) quienes demuestran un nivel regular en la lectura comprensiva, lo cual involucró al pensamiento crítico cuyo $73,6 \%$ evidenció una aplicación de a veces. Con ello, se evidencia los resultados en el nivel lector no son los suficientes para reforzar el pensamiento crítico.

La hipótesis específica 1 se confirma una relación moderada de 0,542 debido a los resultados de mayoría de la muestra, en donde la lectura comprensiva es regular $(74,3 \%)$, en especial en el nivel literal, mientras que la búsqueda de la verdad es a veces aplicado por el $77,9 \%$. Entonces, alertamos que el nivel lector de estudiante no resulta suficiente para emitir juicios con fundamentos.

En la hipótesis específica 2 se evidencia una relación buena de 0,655 entre la lectura comprensiva con un nivel regular por un $74,3 \%$, presente en las categorías literal, inferencial y criterial; y la amplitud mental es aplicado a veces por un $60,0 \%$. Por ello, se expresa que los niveles lectores universitarios no son suficientes para realizar un adecuado debate con personas con puntos de vista diferentes.

En el tercer planteamiento, se demostró una relación de 0.508 entre la lectura comprensiva y la capacidad de análisis, los cuales se deben al resultado mayoritario de la primera variable considerado por un $74,3 \%$ de regular, en tanto que la capacidad de análisis es visto por el $57.9 \%$ como a veces. Es decir, sus capacidades lectoras al no tener un nivel superior consiguen que no desarrolle su capacidad de análisis y uso de la razón en temas complejos.

En el cuarto planteamiento, se obtuvo un nivel relacional buena de 0,608 entre la lectura comprensiva y el ser sistemático, cuyo análisis se debe la mayoría representado por $74,3 \%$ categoriza la variable $X$ como regular, mientras que el $65,7 \%$ considera de a veces el ser sistemático. En otras palabras, sus capacidades para comprender un texto también se ven limitadas por la falta de práctica en la organización de ideas y puntos de vista con un alto grado de meticulosidad.

En la hipótesis específica 5 se encontró una relación moderada de 0582 entre la lectura comprensiva cuya mayoría lo categoriza de regular $(74,3 \%)$ y la confianza en la razón considerada por el $52,9 \%$ como a veces. Por ello, las capacidades para la comprensión de los textos en etapa universitaria se ven disminuidas también en la confianza de sus habilidades de razonamiento. 
En la hipótesis específica 6 se identificó una relación buena de 0,664 entre la lectura comprensiva con un nivel regular por el $74,3 \%$ de estudiantes y la curiosidad es aplicado a veces por un 58,6\%. Por ello, se expresa que los niveles lectores universitarios no son suficientes para reforzar su interés para adquirir conocimientos lejos de pasar un determinado curso.

En el planteamiento siete se identificaron una relación moderada de 0,667 entre la lectura compresiva y la madurez para formular juicios, los cuales se deben al resultado mayoritario de la primera variable considerado por un $74,3 \%$ de regular, en tanto que la madurez para formular juicios es visto por el 58,6\% como a veces. Es resumen, sus capacidades lectoras al no tener un nivel superior disminuyen su frecuencia para reflexionar sobre sus puntos de vista para brindar soluciones.

Realizando un contraste de resultados con estudios anteriores, en donde Salas (2012) identificó que los estudiantes presentan problemas para comprender textos debido a que no practican de forma frecuente las estrategias para ello, además de tener un limitado conocimiento léxico y semántico.

En cuanto al pensamiento crítico tenemos a Perea (2017) señala que el estudiante este motivado e interesado en investigar para desarrollar su pensamiento crítico, aunque no tocamos de forma precisa la motivación, consideramos que la curiosidad y la investigación, conseguido mediante la lectura ayudan a incrementar y fortalecer los criterios del estudiante sobre un tema específico.

\section{CONCLUSIONES}

1. Se evidencia que el nivel lector de los estudiantes de la muestra resulta insuficiente para desarrollar el pensamiento crítico.

2. Concluimos que las dificultades lectoras se presentan en sus calificaciones, por ello limitan al desarrollo de la búsqueda de la verdad que no se manifiesta con una frecuencia aceptable.

3. Los niveles lectores del estudiante universitario impide que estos manejen un debate con personas con puntos de vista diferentes.

4. En conclusión, las dificultades lectoras se presentan en sus calificaciones, por ello limitan al desarrollo de su capacidad de análisis y uso de la razón en temas complejos.

5. Existe una relación buena entre la lectura comprensiva (regular) y el ser sistemático (a veces) cuyas categorizaciones intermedias evidencian que las dificultades dominar los distintos tipos de lectura, lo cual limita su capacidad para organizar sus ideas y puntos de vista con un alto grado de meticulosidad.

6. Las dificultades lectoras se presentan en sus calificaciones, lo cual se evidencia en la confianza de sus habilidades de razonamiento.
7. El nivel lector de los estudiantes de la muestra resulta insuficiente para adquirir conocimientos lejos de pasar un determinado curso.

8. Las dificultades lectoras se presentan en sus calificaciones, disminuyen la posibilidad de reflexionar sobre sus puntos de vista para brindar soluciones.

\section{AGRADECIMIENTOS}

Mi sincero agradecimiento a la UNASAM, especialmente a los estudiantes de la FCM y al Dr. Abelardo R. Campana Concha por las orientaciones brindadas para el desarrollo del presente artículo.

\section{REFERENCIAS BIBLIOGRÁFICAS}

Cangalaya, L. (2020). Habilidades del pensamiento crítico en estudiantes universitarios a través de la investigación. Desde el Sur, 12 (1). http://www.scielo.org.pe/scielo.php?pid=S2415$09592020000100141 \&$ script=sci_arttext

Córdova, D. (2019). Estrategias metodológicas y la comprensión lectora de textos expositivos en estudiantes de 1er. Grado de Educación Secundaria de la I.E. Fe y Alegría N49 Paredes Maceda - Ventiséis De Octubre, Piura [Tesis de Maestría, Universidad de Piura].

Mendoza, P. (2015). La investigación y el desarrollo de pensamiento crítico en estudiantes universitarios [Tesis Doctoral, Universidad de Málaga ]. https://riuma.uma.es/xmlui/bitstream/handle/10630/118 83/TD_MENDOZA_GUERRERO_Pedro_Luis.pdf?sequ ence $=1$

Ministerio de Educación (2018). Evaluación PISA 2018 [ A r c h i v o P D F ]. https://es.calameo.com/read/006286625977c1ced4d6c ?view=slide \&page $=4$

Perea, L. (2017). Disposición hacia el pensamiento crítico y su relación con el rendimiento académico en estudiantes del curso de Metodología de la Investigación de una Universidad Privada de Lima Metropolitana [Tesis de Maestría, Universidad Peruana Cayetano Heredia].

Pineda, M. y Cerrón, A. (2015) Pensamiento crítico y rendimiento académico. Revista Horizonte de la ciencia, $5 \quad(8), \quad 105-110$. https://revistas.uncp.edu.pe/index.php/horizontedelacie ncia/article/view/299/312

Salas, P. (2012). El desarrollo de la comprensión lectora en los estudiantes del tercer semestre del nivel medio superior de la Universidad Autónoma de Nuevo León [Tesis de Maestría, Universidad Autónoma de Nuevo León]. http://eprints.uanl.mx/3230/1/1080256466.pdf 